\title{
Efecto de plantaciones de Pinus radiata y Eucalyptus globulus sobre el recurso agua en la Cordillera de la Costa de la región del Biobío, Chile
}

\author{
Effect of Pinus radiata and Eucalyptus globulus plantations on water resource \\ in the Coastal Range of Biobío region, Chile \\ Anton Huber ${ }^{a *}$, Andrés Irouméb ${ }^{b}$ Christian Mohrc ${ }^{c}$ Cristian Frêne ${ }^{a}$ \\ *Autor de correspondencia: aUniversidad Austral de Chile, Facultad de Ciencias, Valdivia, Chile, \\ tel.: 56-63-221359, ahuber@uach.cl \\ bUniversidad Austral de Chile, Facultad de Ciencias Forestales y Recursos Naturales, Valdivia, Chile. \\ cUniversity of Potsdam, Institute for Geoecology, Hydrology and Climatology, Potsdam, Germany.
}

\begin{abstract}
SUMMARY
Rainfall redistribution, temporal oscillation of streamflow, variation of soil water content, evapotranspiration and sediment transport of two Pinus radiata and two Eucalyptus globulus catchments were determined. The catchments are located in the Coastal Range of southern central Chile. Due to their age, these plantations are to be harvested soon. The P. radiata plantations registered an interception loss of 17 and $16 \%$, values surpassing significantly the eucalyptus values of 10 and $11 \%$. The streamflows of the same catchments were 705 resp. $707 \mathrm{~mm}$ and 439 resp. $500 \mathrm{~mm}$ for the monitored period of 14 months. Despite the higher amount of rainfall reaching the soil in the eucalyptus-catchments, these catchments registered low streamflow compared to the pine-catchments. During the dry summer, all soils showed a severe reduction of their soil water content, exceeding the permanent wilt point in the soil's upper $30 \mathrm{~cm}$. The E. globulus-catchments registered an evapotranspiration equal to 1,582 and 1,469 mm while it reached values of 1,357 and 1,298 in the P. radiata-catchments. These values correspond, for the E. globulus-catchments, to 74 and $68 \%$ of total precipitation and 63 and $60 \%$ for the $P$. radiata-catchments. These differences may be higher if water availability during summer drought does not limit the total evapotranspiration. The sediment export reached values of 237 and $615 \mathrm{~kg} / \mathrm{ha}$ for the P. radiata-catchments and 152 and $125 \mathrm{~kg} / \mathrm{ha}$ for the E. globulus-catchments during the monitored period.
\end{abstract}

Key words: streamflow, water balance, sediment transport, catchments, Pinus radiata, Eucalyptus globulus.

\section{RESUMEN}

Se estudió el efecto de plantaciones forestales sobre el recurso hídrico en cuencas de la vertiente este de la Cordillera de la Costa de la región del Biobío, en el centro sur de Chile. La redistribución de las precipitaciones, la oscilación temporal de los caudales, la fluctuación del contenido de agua edáfica, la evapotranspiración y el transporte de sedimentos fueron determinados para dos cuencas forestadas con Pinus radiata y dos con Eucalyptus globulus. De los $2.149 \mathrm{~mm}$ de precipitación registrada, las plantaciones de $P$. radiata interceptaron el 16 y $17 \%$, y las de E. globulus un 10 y $11 \%$, respectivamente. El caudal de los efluentes para las dos cuencas con $P$. radiata fue equivalente al $33 \%$, monto que superó a las con E. globulus que alcanzaron el 20 y $23 \%$ de la precipitación. Durante el verano, los suelos presentaron una fuerte disminución de su contenido de agua, sobrepasando en los primeros $30 \mathrm{~cm}$ el punto de marchitez permanente. Las cuencas con E. globulus tuvieron una evapotranspiración equivalente al 76 y $70 \%$, y las de $P$. radiata al 65 y $64 \%$, respectivamente, de la precipitación. Estas diferencias pudieron ser superiores si la disponibilidad de agua, durante el estío, no hubiese sido una limitante para satisfacer los requerimientos máximos de agua de cada una de las especies. La exportación total de sedimentos para el período fue de 237 y $615 \mathrm{~kg} / \mathrm{ha}$ en las cuencas con $P$. radiata y 152 y $125 \mathrm{~kg} / \mathrm{ha}$ con E. globulus. Los resultados obtenidos siguen una tendencia semejante a los de investigaciones en estudios con condiciones edafoclimáticas y plantaciones similares.

Palabras clave: balance hídrico, transporte sedimentos cuencas, Pinus radiata, Eucalyptus globulus.

\section{INTRODUCCIÓN}

Hay opiniones diversas con respecto al real impacto que ocasionan las cubiertas forestales en el régimen hidrológico y en la magnitud de la erosión (Robinson et al. 2003, Andreassian et al. 2004, Boothroyd et al. 2004, Brown et al. 2005, Gomi et al. 2005, Farley et al. 2005, Hassan et al. 2005, Iroumé et al. 2006, Hubbart et al. 2007, Huber et al. 2008, Scott y Prinsloo 2008). En general, estos autores concuerdan que, luego de unos años del establecimiento de una plantación, los caudales comienzan a disminuir debido a un aumento de la tasa evapotranspirativa. Luego de la cosecha final, los caudales recuperan los niveles que tenían al inicio y se incrementan las tasas de transporte de sedimento. 
Los bosques en general y las plantaciones forestales en particular involucran una mayor cantidad de agua en la evapotranspiración y registran una superior pérdida de agua por intercepción del dosel en comparación a otros tipos de cubierta vegetal. La magnitud de estos alcances pasa por las características de las plantaciones (especie, edad y manejo), particularidades de las precipitaciones (cantidad e intensidad) y de las condiciones meteorológicas (Crockford y Richardson 2000, Van Dijk y Bruijnzeel 2001, Iroumé y Huber 2002, Carlyle-Moses 2004, Vega et al. 2005, Huber et al. 2008).

El transporte de sólidos suspendidos tiene un rol fundamental en el ciclo biogeoquímico de las cuencas forestadas (Karwan et al. 2007). Ellos degradan el hábitat acuático y rompen las conexiones en la relación sueloagua, aumentan el transporte de contaminantes adsorbidos a las partículas e incrementan los costos de tratamiento asociados al uso público del agua (Rehg et al. 2005, Gomi et al. 2005). Las perturbaciones ocasionadas por el manejo de las plantaciones están relacionadas con un incremento en la producción de sedimentos suspendidos (Megahan et al. 1995, Karwan et al. 2007, Hubbart et al. 2007, May 2007), lo que implica importantes desafíos para la relación entre el manejo del bosque y la calidad de agua (Karwan et al. 2007).

Las unidades geomorfológicas más degradadas en la zona centro-sur de Chile están ubicadas en la vertiente oriental de la Cordillera de la Costa, en el llamado secano interior, especialmente las correspondientes a la región del Biobío. En tiempos pasados, esta zona estuvo cubierta por bosque nativo (Armesto et al. 2010) que fue intensamente explotado, dejando en muchos casos el suelo expuesto a la erosión. Actualmente, la mayor parte de esta superficie posee una cubierta herbácea, arbustiva, plantaciones forestales o se está usando en una actividad agrícola-ganadera precaria. La degradación de los suelos es más evidente en laderas con mayor pendiente y en suelos muy alterados debido a intervenciones silviculturales precedentes. El creciente deterioro de estos suelos restringe su uso a la actividad forestal, preferentemente a la forestación con especies exóticas de rápido crecimiento, que registra un notorio incremento en las últimas décadas. En la región del Biobío la superficie plantada con Pinus radiata D. Don (pino) y Eucalyptus spp. supera las 610.000 y 240.000 ha, respectivamente (INFOR 2008).

Si bien en Chile se ha realizado varios estudios sobre el consumo de agua de ambas especies (Huber et al. 1985, 1998, 2008, Oyarzún et al. 1985, Huber y García 1999, Huber e Iroumé 2001, Huber y Trecaman 2002, 2004, Iroumé et al. 2006), son escasos los antecedentes que indican la magnitud de los procesos erosivos que se registran en estas superficies forestadas (Oyarzún 1993, Oyarzún y Peña 1995, Pizarro y Cuitiño 1999).

Este trabajo pretende aportar al conocimiento sobre el impacto que tienen las plantaciones forestales sobre el recurso agua en la zona centro-sur de Chile. Las hipótesis de esta investigación son que: a) las distintas características que tiene el dosel de las plantaciones forestales afectan las pérdidas de agua por intercepción y, por consiguiente, la cantidad total de agua que alcanza el suelo en cada cuenca; b) que los desiguales montos de agua involucrados en la evapotranspiración repercuten sobre el caudal de los efluentes y c) que las disimilitudes entre los valores de los diferentes componentes del balance hídrico y las particularidades de las cuencas definen la cantidad de sedimentos transportados. Para probar estas hipótesis, se plantean como objetivos específicos el confrontar la dinámica hídrica y el transporte de sedimentos por los efluentes de microcuencas forestadas con Pinus radiata y Eucalyptus globulus Labill (eucalipto), ubicadas en la vertiente este de la Cordillera de la Costa, en la región del Biobío, sur de Chile.

\section{MÉTODOS}

Características del área de estudio. Para el estudio se seleccionaron cuatro microcuencas hidrográficas ubicadas a $3 \mathrm{~km}$ al oeste de la ciudad de Nacimiento, región de Biobío, Chile (latitud $37^{\circ} 28^{\prime \prime} \mathrm{S}$, longitud $72^{\circ} 42^{\prime \prime} \mathrm{O}$ ), que pertenecen a la empresa Forestal Mininco S. A. (figura 1).

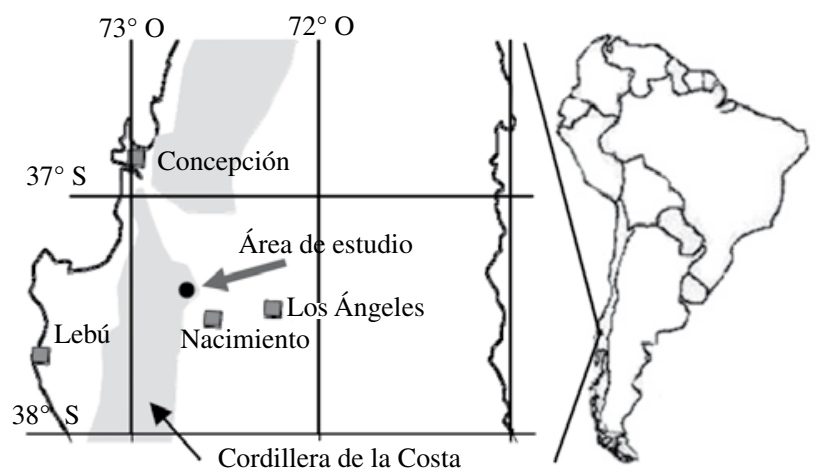

Figura 1. Localización de las cuencas forestales en Nacimiento, Chile.

Location of the forested catchments in Nacimiento, Chile.

Las cuencas están ubicadas en la vertiente este de la Cordillera de la Costa, distantes a menos de $1,5 \mathrm{~km}$ entre sí. Dos de estas cuencas están forestadas con Pinus radiata y las otras, con Eucalyptus globulus, todas en víspera de alcanzar la edad de rotación (cuadro 1). Los cauces de las cuencas se localizan en barrancos que se han labrado por la acción erosiva del agua a través del tiempo. La pendiente media supera el $20 \%$, la que le otorga una importante torrencialidad durante la época más lluviosa del año.

La zona posee un clima de tipo templado infratermal estenotérmico con un régimen mediterráneo perhúmedo (Fuenzalida 1971). La precipitación promedio anual de la zona es de $1.150 \mathrm{~mm}$ (INIA 1989) y se concentra de abril a septiembre. Posee una estación seca corta en verano (menos de cuatro meses) durante los cuales no llueve más del $5 \%$ del total anual. 
Cuadro 1. Características de las cuencas hidrográficas con Pinus radiata (pino) y Eucalyptus globulus (eucalipto). Hydro-geomorphologic characteristics of the Pinus radiata and Eucalyptus globulus catchments.

\begin{tabular}{lcccc}
\hline \multirow{2}{*}{ Característica } & \multicolumn{3}{c}{ Cuenca } \\
\cline { 2 - 5 } & Pino 1 & Pino 2 & Eucalipto 1 & Eucalipto 2 \\
\hline Área de la cuenca (ha) & 12,55 & 13,98 & 16,72 & 21,1 \\
Longitud cauce principal (km) & 0,47 & 0,41 & 0,45 & 0,62 \\
Pendiente media (\%) & 25,2 & 30,3 & 27,7 & 36,5 \\
Pendiente media del cauce (\%) & 22 & 24 & 26 & 27 \\
Perímetro (km) & 1,7 & 1,57 & 2,0 & 2,1 \\
Índice de Gravelius & 1,35 & 1,18 & 1,37 & 1,25 \\
Altitud media (m) & 327 & 331 & 369 & 320 \\
Coeficiente orográfico $\left(\mathrm{m}^{2} / \mathrm{ha}\right)$ & 85,01 & 78,44 & 81,48 & 48,51 \\
Densidad de drenaje $\left(\mathrm{km} / \mathrm{km}^{2}\right)$ & 4,67 & 4,40 & 5,91 & 6.67 \\
Canal de alimentación $\left(\mathrm{km} / \mathrm{km}^{2}\right)$ & 0,21 & 0,23 & 0,17 & 0,15 \\
Alejamiento medio $(\mathrm{km})$ & 1,34 & 1,09 & 1,11 & 1,35 \\
Coeficiente de torrencialidad & 37,16 & 31,48 & 35,33 & 31,63 \\
\hline
\end{tabular}

Los eventos de lluvia de esta zona tienen diferente duración, pudiendo prolongarse hasta por varios días. Sus intensidades rara vez sobrepasan el equivalente a los $20 \mathrm{~mm} / \mathrm{h}$. Las precipitaciones de la zona son preferentemente del tipo ciclonal o frontal. Registran considerables diferencias interanuales en su cuantía y su distribución local está influida por la topografía de la cordillera. Durante los eventos de lluvia, y debido a la ubicación altitudinal de las cuencas, las plantaciones se encuentran, generalmente, inmersas en neblina o nubes.

La temperatura promedio anual del aire es de $13{ }^{\circ} \mathrm{C}$ y oscila entre los $7^{\circ} \mathrm{C}$ julio y $19^{\circ} \mathrm{C}$ enero. Durante el estío, en días con alta radiación solar, se pueden registrar temperaturas máximas que sobrepasan los $35^{\circ} \mathrm{C}$. Debido al rápido ascenso del estado térmico del aire, la humedad relativa puede descender a valores inferiores al $20 \% 1$. Estas condiciones meteorológicas propician una alta evapotranspiración potencial.

La zona tiene suelos derivados de rocas metamórficas cuya textura está influida por su evolución y erosión. Son suelos franco-arenosos a arcillo-limosos en la superficie y en profundidad son franco a arcillosos, con drenaje interno moderado (Schlatter et al. 2003). En los sitios de estudio, el suelo tiene profundidades variables que fluctúan entre 0,5 y $3 \mathrm{~m}$.

La velocidad de infiltración ${ }^{2}$ de estos suelos, en estado saturado, oscila entre 3 y $10 \mathrm{~mm} / \mathrm{h}$. La dispersión de estos valores se debe principalmente a la heterogénea estructura de los suelos, que contienen una considerable cantidad de piedras y de rocas fragmentadas de diferentes tamaños.

Registros realizados durante el estudio con termohigrógrafos. Determinados con infiltrómetros de doble anillo (Steubing y Fangmeier 2001).
A ello se agrega la compleja distribución espacial de las raíces de la vegetación herbácea, arbustiva y de las plantaciones forestales anteriores y actuales, además de las perturbaciones a las que estuvieron expuestos durante las actividades forestales precedentes. Estas características no son un obstáculo importante a la humectación en profundidad del suelo, considerando la intensidad de las lluvias en la zona de estudio.

Las características de las dos plantaciones de pino (Pino 1 y Pino 2) se presentan en el cuadro 2. Ellas fueron establecidas en 1987, la densidad inicial de las plantaciones fue de 1.250 árboles/ha y corresponden a una segunda rotación. En la fecha del estudio tenían 23 años de edad y, por consiguiente, estaban prontas a ser cosechadas. Presentaban dos raleos y una poda hasta los $6 \mathrm{~m}$ de altura.

Cuadro 2. Características de las plantaciones de Pinus radiata (pino) y Eucalyptus globulus (eucalipto).

Characteristics of the Pinus radiata and Eucalyptus globulus plantations.

\begin{tabular}{lcccc}
\hline & \multicolumn{4}{c}{ Cuenca } \\
\cline { 2 - 5 } Característica & Pino 1 & Pino 2 & Eucalipto Eucalipto \\
& & & 1 & 2 \\
\hline Edad (años) & 23 & 23 & 9 & 9 \\
Altura media (m) & 28,2 & 28,2 & 20,4 & 20,3 \\
Densidad (árboles/ha) & 320 & 315 & 1.174 & 1.326 \\
DAP medio (cm) & 36,0 & 35,4 & 15,9 & 15,2 \\
Cobertura copa (\%) & 65 & 65 & 50 & 55 \\
Altura poda (m) & 5,9 & 5,7 & Sin poda & Sin poda \\
Área basal (m $\left.{ }^{2} / \mathrm{ha}\right)$ & 32,9 & 31,7 & 24,3 & 25,2 \\
Zona buffer (ha) & 1,5 & 0,9 & 0,8 & 1,0 \\
\hline
\end{tabular}


El suelo de estas plantaciones estaba cubierto por un mantillo dominado por una capa de acículas que, en promedio, no sobrepasaba 2 a $3 \mathrm{~cm}$ de espesor, pero en sus microdepresiones con posibilidades de acumulaciones alcanzó hasta $8 \mathrm{~cm}$ de espesor. La cubierta herbácea era rala $(<25 \%)$ y estaba conformada principalmente por gramíneas que, habitualmente, perecen durante el estío por estrés hídrico. La cubierta arbustiva era dispersa, conformada mayoritariamente por maqui (Aristotelia chilensis Mol.), zarzamora (Rubus radicans Cav.), además de algunas especies arbóreas todavía de baja altura como litre (Lithraea caustica Mol.), boldo (Peumus boldus Mol.), peumo (Cryptocarya alba Mol.), arrayán (Luma apiculata DC. Burret.), avellano (Gevuina avellana Mol.) y ocasionalmente roble (Nothofagus obliqua (Mirb.) Oerst.).

El efluente de la cuenca de Pino 1 poseía en toda su extensión una zona de protección con un ancho de $13 \mathrm{~m} \pm 4 \mathrm{~m}$. En la cuenca Pino 2, la zona de protección tenía un ancho de $11 \mathrm{~m} \pm 3 \mathrm{~m}$. El estrato arbustivo y arbóreo de estas zonas presentaba una cobertura que fluctuaba entre 50 y $70 \%$, conformada por quila (Chusquea quila Mol.), zarzamora, maqui, lingue (Persea lingue Nees.), arrayán, avellano, boldo y roble. La cobertura vegetacional herbácea era baja $(<25 \%) \mathrm{y}$, en conjunto, todos los estratos generaban una cobertura $>75 \%$.

Las particularidades de las dos plantaciones de eucalipto se presentan en la cuadro 2. Eucalipto 1 era una plantación de segunda rotación establecida en 1999. Eucalipto 2 tenía la misma edad pero de monte bajo, es decir, de regeneración por tocón, y cada tocón presentaba entre dos y tres fustes. El suelo de ambas cuencas presentaba un mantillo homogéneo que no sobrepasa $2 \mathrm{~cm}$ de espesor. La cubierta arbustiva era rala, conformada principalmente por retamillo (Teline monspessulana (L.) Koch) y algunos individuos de mosqueta (Rubus ulmifolius Schott f.). La vegetación herbácea era escasa y estaba conformada por algunas gramíneas que perecen durante el verano.

El efluente de la cuenca Eucalipto 1 presentaba en toda su extensión una zona de protección ripariana de una sección total de $41 \mathrm{~m} \pm 6 \mathrm{~m}$. En la parte baja de la microcuenca, cercana al vertedero, se observó una pequeña superficie anegada. El estrato arbustivo y arbóreo de esta zona presentó una cobertura cercana al $75 \%$, conformado por zarzamora, quila, maqui y retamillo, con presencia ocasional de algunos individuos arbóreos de tamaño menor de lingue, arrayán, peumo, roble y algunos árboles de pino. También se observaron algunos canelos (Drimys winteri Forster) y nalcas (Gunnera tinctoria (Mol.) Mierbel.) en la zona anegada. La cobertura herbácea era baja $(<25 \%)$, conformada principalmente por enredaderas quilineja (Luzuriaga radicans Ruiz et Pavón.) y voqui (Boquila trifoliolata (DC) Decne), además de helechos (Lophosoria quadripinnata (Gmel.) Chr). El piso estaba cubierto por un mantillo fraccionado de hojas de eucalipto, con espesor promedio de $2 \mathrm{~cm}$. En conjunto, todos los estratos generaban una cobertura $>75 \%$.
El efluente de la cuenca Eucalipto 2 también presentaba una protección ripariana con un ancho de $52 \mathrm{~m} \pm 8 \mathrm{~m}$ en toda su extensión. El estrato arbustivo y arbóreo de esta franja mostraba una cobertura cercana al $60 \%$, conformada por zarzamora, quila, maqui y murta (Ugni molinae Turcz.), con presencia ocasional de boldo, lingue, peumo, roble, piñol (Lomatia dentata (Ruiz et Pavón) Br.), naranjillo (Citronella mucronata (Ruiz et Pavón) y chupón (Greigia sphacelata (Ruiz et Pavón) Regel). La cobertura herbácea era baja $(<25 \%)$ y el piso estaba cubierto por un mantillo de hojas de eucalipto con espesor promedio de $3 \mathrm{~cm}$. En conjunto, los diferentes estratos generaban una cobertura cercana al $65 \%$.

Aportes de agua. La cantidad de precipitación que llegó a las cuencas se determinó con dos pluviógrafos (Hobo®), ubicados a campo abierto, cerca de ellas. La cantidad total de agua que alcanzó el piso (precipitación neta) se fraccionó en precipitación directa y escurrimiento fustal [1].

$$
P n=P d+P f
$$

Donde $P n=$ precipitación neta $(\mathrm{mm}), P d=$ precipitación directa $(\mathrm{mm})$ y $P f=$ escurrimiento fustal $(\mathrm{mm})$.

Debido a la especial distribución temporal de las precipitaciones, el período de mediciones se tuvo que extender hasta septiembre 2009. Ello fue necesario para que los suelos, al final de este lapso de tiempo, alcanzaran un contenido de agua similar al que tuvieron al inicio. Esta condición es requisito indispensable para calcular la cantidad de agua involucrada en la evapotranspiración con la ecuación [4] que se presenta más adelante.

Las pérdidas de agua por intercepción se consideraron equivalentes al diferencial entre la precipitación total registrada a campo abierto y la precipitación neta [2].

$$
I c=P p-P n
$$

Donde $I c=$ pérdidas de agua por intercepción $(\mathrm{mm}) \mathrm{y}$ $P p=$ precipitación $(\mathrm{mm})$.

Para lograr esta partición se delimitó, en cada cuenca, una parcela de $15 \times 40 \mathrm{~m}$. Las precipitaciones que alcanzaron el suelo (precipitación directa) se determinaron con una canaleta metálica en forma de $\mathrm{V}$, de $15 \mathrm{~cm}$ de ancho y $30 \mathrm{~m}$ de largo, instalada a $30 \mathrm{~cm}$ sobre el suelo. La fracción de las precipitaciones que llegó al suelo, utilizando como senda de fluido el fuste de los árboles (escurrimiento fustal), fue recogida por collarines de goma sellados alrededor del tronco de 15 árboles según la metodología propuesta por Huber y Oyarzún (1984). El agua recogida, en cada caso, fue conducida a su respectivo registrador.

Para establecer si existieron diferencias significativas entre las pérdidas de agua por intercepción de las diferentes 
cuencas, se confrontaron los valores de estas mermas calculados para cada uno de los eventos de lluvia con la prueba Mann-Whitney.

Escorrentía. En los efluentes, a la salida de cada una de las cuencas, se construyó un vertedero de concreto armado tipo Thompson de paredes delgadas. Esta construcción tiene $3 \mathrm{~m}$ de largo, 1,5 m ancho y 1,5 m de alto con una escotadura de $60^{\circ}$, cuyo vértice estaba ubicado a $50 \mathrm{~cm}$ sobre su piso. Debido a la conformación pedregosa de la base de los cauces, las construcciones fueron insertadas a $50 \mathrm{~cm}$ de profundidad en el lecho del efluente para prevenir filtraciones subterráneas. Debido a esta necesidad, a lo ancho de toda la entrada de los vertederos se construyó un muro de $50 \mathrm{~cm}$ de alto. Esta obra transformó a los vertederos en receptáculos con una superficie de $4,5 \mathrm{~m}^{2}$. Estas estructuras de cemento se aprovecharon como trampas para retener los sedimentos de arrastre y, simultáneamente, como colectores de los sedimentos en suspensión que se decantaron. En la pared frontal de los vertederos, a $20 \mathrm{~cm}$ de cada pared lateral y a la altura de la base de su piso, se empotraron dos compuertas de hierro de $30 \mathrm{~cm}$ de ancho y 20 de alto. Estas cerraduras se abrieron durante la limpieza de los vertederos, para facilitar la evacuación del agua y del material depositado en el fondo después de terminar los controles periódicos. Para determinar el caudal de los efluentes se instaló una estación fluviométrica en cada vertedero. Estos equipos funcionan con el principio del flotador. Fueron construidos en la Universidad Austral de Chile y tienen una precisión de $2 \mathrm{~mm}$. Cada vertedero fue calibrado en terreno a través de aforos periódicos para cubrir un amplio rango de caudales y lograr con ello la correspondiente curva de aforo (ecuación polinomial de grado tres, $\left.\mathrm{R}^{2}=0,999, P<0,01\right)$. La frecuencia de registro se fijó en tres minutos.

Con esta información se determinó la variación temporal del caudal de cada efluente. Se realizaron comparaciones estadísticas con los valores horarios de los montos de escorrentía entre las cuencas mediante una prueba no paramétrica de comparaciones de pares U de Mann-Whitney. Todos los análisis estadísticos fueron desarrollados utilizando el programa R para Windows. En todos los análisis estadísticos las diferencias entre tratamientos se consideraron significativas cuando $P \leq 0,05$.

También se determinaron los coeficientes de escorrentía de cada cuenca por simple relación entre la escorrentía total del período de estudio y la precipitación para el mismo lapso de tiempo.

Para cada evento de lluvia que se manifestó en el hidrograma se determinó la fracción de la componente de escorrentía superficial que participó en el caudal total. Para esta partición se utilizó el método de la pendiente constante. A su vez, para cada cuenca, se determinó la variación periódica de la participación de la escorrentía superficial en el caudal total de los efluentes.
Transporte de sedimentos. Para determinar la cantidad de sedimentos en suspensión que pasó por cada vertedero se construyó una estructura rectangular flotante $(50 \times 30 \mathrm{~cm})$ con tubos de PVC de $5 \mathrm{~cm}$ de diámetro. A esta armazón, en su zona central, se fijó una bomba eléctrica de $12 \mathrm{~V}$, cuya bocatoma permaneció sumergida a $5 \mathrm{~cm}$ de profundidad. Esta localización se pudo mantener constante porque la estructura PVC estaba articulada a dos brazos móviles de 1,5 m de largo, fijados en la parte alta, al final de cada una de las paredes laterales del vertedero.

Para obtener muestras homogéneas, integradas y representativas de agua de los efluentes, la bomba movilizaba un volumen que siempre estaba en relación directa al caudal del efluente en el momento de la extracción. El agua de cuatro muestras diarias y para un período aproximado de siete días se almacenó en un recipiente. A esta agua se le determinó la concentración de sedimentos, utilizando un sistema de filtración con bomba de vacío. Se usaron filtros de fibra de vidrio (Advantec GF75 $47 \mathrm{~mm}$ ) que fueron secados a $60{ }^{\circ} \mathrm{C}$ por 48 horas. Este valor se multiplicó por el caudal del correspondiente período para establecer la carga de sedimentos en suspensión que fue evacuada por el efluente.

Periódicamente se cuantificaron los sedimentos acumulados en el fondo de los vertederos. Para ello se diseñó y construyó un artefacto que consiste en un disco de hierro de $8 \mathrm{~cm}$ de diámetro y $1 \mathrm{~mm}$ de espesor que, en su cara superior, tiene adosada una lámina delgada de goma. En el centro de la cara superior del disco de hierro tiene soldada una varilla de hierro de $5 \mathrm{~mm}$ de diámetro y 1,5 m de largo, cuyo extremo termina en un hilo 4,8 mm (3/16"). Seis de estas estructuras se distribuyeron en forma sistemática y equidistante en cada vertedero mediante sujeciones que las separaban a $40 \mathrm{~cm}$ de cada una de las paredes laterales.

Para establecer la cantidad de sedimentos que se depositaron sobre cada disco se sobrepuso a la varilla de hierro un tubo de acero de $4 \mathrm{~cm}$ de diámetro, que, en uno de sus extremos, tiene un borde cortante que hace contacto con la goma del disco de hierro. En la otra punta está soldado un mango que permite girar y presionar con fuerza el tubo contra la goma para lograr un buen cierre. Para mantener su equidistancia a la varilla y, por consiguiente, asegurar una ubicación concéntrica sobre el disco, el tubo tiene en su interior una guía flotante. Logrado este posicionamiento y contacto, el tubo de $4 \mathrm{~cm}$ de diámetro se presionaba en forma hermética contra el disco con una pieza que se atornillaba al hilo de la varilla de hierro. Posteriormente, se sacaba completamente el artefacto del vertedero y se transvasaba su contenido a un recipiente. Las muestras fueron secadas a $60^{\circ} \mathrm{C}$. El peso del material sólido anhidro se consideró como la cantidad de sedimentos que estuvieron depositados sobre la superficie del disco, delimitada por el tubo de $4 \mathrm{~cm}$ de diámetro menos el área ocupado por la varilla de hierro. Con esta metodología se determinó la cantidad de sedimentos que hubo por unidad de superficie 
en cada uno de los discos. El valor promedio obtenido de los seis discos se extrapoló al área del piso del vertedero para calcular la cantidad total de sedimentos depositados en él. Debido a que en este caso sólo se contó con información semanal, se utilizó en el análisis estadístico mediante prueba no paramétrica de comparaciones de pares de Kruskal-Wallis entre las cuencas.

Agua edáfica y evapotranspiración. Aproximadamente cada 30 días se determinó la variación temporal y espacial del contenido de agua del suelo hasta los 2,5 m de profundidad. Para ello se hicieron mediciones con un equipo DTR (TRIME-FM3, sonda T3, Field Measurement Device Version P3 marca IMKO). Estos registros se hicieron en 10 puntos repartidos homogéneamente en superficie en cada una de las cuencas, con una secuencia en profundidad de $10 \mathrm{~cm}$.

Para determinar la cantidad de agua involucrada en la evapotranspiración de las cuencas se utilizó la ecuación [3].

$$
E v T r=P p-Q-\Delta R-\Delta F
$$

Donde $E v T r=$ evapotranspiración, $P p=$ precipitaciones, $Q=$ escorrentía, $\Delta R=$ cambio en el almacenamiento de agua en el suelo y $\Delta F=$ cambio en almacenamiento de aguas subterránea (freática).

Como no se pudo determinar la variación periódica del contenido de agua subterránea, tampoco fue posible inferir la variación temporal de la evapotranspiración. En consecuencia, la cantidad de agua involucrada en la evapotranspiración sólo se pudo establecer para el período completo, al suponer que el contenido de agua subterránea al inicio del período de estudio fue similar a la del final $(\Delta F=0)$. En consecuencia, la ecuación original [3] se redujo a la ecuación [4].

$$
E v T r=P p-Q-\Delta R
$$

\section{RESULTADOS}

Aportes de agua. La precipitación total del período (15 junio 2008-30 septiembre 2009) fue de $2.149 \mathrm{~mm}$ (cuadro 3). La mayor parte de las lluvias se registraron entre los meses de mayo y septiembre, mientras que los meses correspondientes al estío fueron excepcionalmente deficitarios en precipitaciones (cuadro 3).

En las cuencas con pino la confrontación de los valores de intercepción correspondientes a cada uno de los eventos de lluvia no registró diferencias estadísticas $(P=0,846)$, similar situación sucedió entre las cuencas con eucaliptos $(P=0,370)$ (cuadro 3$)$. La plantación de Pino 1 tuvo una mayor intercepción que Eucalipto $1 \mathrm{y}$ $2(P<0,05$ y $P<0,01)$, semejante resultado se registró entre Pino 2 y las dos cuencas con eucalipto $(P<0,01$ y $P<0,001)$.
Escorrentía. El coeficiente de escorrentía para las dos cuencas con pino fue del $33 \%$ y para las de Eucalipto 1 y 2 del 20 y $23 \%$, respectivamente (cuadro 3). Las cantidades diarias de agua que pasaron por los vertederos de las cuencas forestadas con pino durante todo el período no tuvieron diferencias significativas $(P=0,680)$. Diferente fue la situación entre Eucalipto 1 y $2(P<0,05)$. También hubo diferencias significativas entre Pino 1 y Eucalipto 1 $(P<0,01)$ y entre Pino 2 y Eucalipto $1(P<0,01)$. La escorrentía total en la cuenca de Pino 1 mostró tendencia a ser mayor que Eucalipto 2, pero las diferencias no fueron significativas $(P=0,588)$. Resultado similar se obtuvo entre las cuencas de Pino 2 y Eucalipto $2(P<0,356)$.

La participación de la componente subsuperficial en el caudal total para las cuencas con Pino 1 y 2 fue del 73 y $74 \%$, respectivamente, y para las cuencas Eucalipto 1 y 2 del 70 y $75 \%$, respectivamente. No hubo diferencias significativas entre los valores periódicos de las cuencas con pino $(P=0,230)$ ni entre las de eucalipto $(P=0,298)$. Similares resultados se obtuvieron entre las cuencas Pino 1 y Eucalipto 1 y $2(P=0,267 ; P=0,305)$ y entre Pino 2 y Eucalipto 1 y $2(P=0,258 ; P=0,317)$.

Agua edáfica y evapotranspiración. La variación espacial y temporal del contenido de agua edáfica para las cuatro cuencas en los primeros 2,5 m de profundidad se presenta en la figura 2 y la variación periódica del contenido total de agua del perfil en el cuadro 3.

La cantidad de agua involucrada en la evapotranspiración total para el período completo de Pino 1 y 2 fue equivalente a 1.405 y $1.388 \mathrm{~mm}$, respectivamente (cuadro 3), que corresponden al 65 y $64 \%$ de la precipitación total, respectivamente. Para Eucalipto 1 y 2 estos valores fueron del 76 y $70 \%$, lo que equivale a 1.630 y $1.511 \mathrm{~mm}$, respectivamente (cuadro 3). Cuando se hicieron estas relaciones entre la cantidad de agua extraída exclusivamente del suelo (evapotranspiración neta) y la cantidad de agua que efectivamente alcanzó el suelo (precipitación neta), los valores disminuyeron para Pino 1 y 2 a un 58 y $56 \%$, y para Eucalipto 1 y 2 al 73 y $66 \%$, respectivamente.

Transporte de sedimentos. La variación temporal de la cantidad total de sedimentos (fondo y en suspensión) para cada una de las cuencas está resumida en el cuadro 3. Los valores periódicos entre las cuencas con pino no presentaron diferencias estadísticas $(P=0,311)$. Tampoco las hubo entre las cuencas plantadas con eucalipto $(P=0,305)$. Resultados semejantes se obtuvieron entre las cuencas Pino 1 y Eucalipto 1 y $2(P=0,291, P=0,301)$ y Pino 2 y Eucalipto 1 y $2(P=0,316$ y $P=0,301)$.

\section{DISCUSIÓN}

Precipitaciones. Las precipitaciones para el período de 14 meses son similares a la suma de las precipitaciones promedio mensuales correspondientes al mismo lapso de 
Cuadro 3. Valores periódicos y totales de la precipitación (Pp), intercepción (Ic), caudal (Q), escorrentía superficial (Es), caudal base $(\mathrm{Cb})$, evapotranspiración (EvTr), contenido de agua edáfica $(0-2,5 \mathrm{~m})(\mathrm{R})$ y transporte total de sedimentos (St) para las cuencas con Pinus radiata (pino, P) y Eucalyptus globulus (eucalipto, E).

Values of total precipitation (Pp), interception loss (Ic), streamflow (Q), event flow (Es), base flow (Cb), evapotranspiration (EvTr), soil water content 0-2,5 m) (R) and total sediment export (St) for Pinus radiata $(\mathrm{P})$ and Eucalyptus globulus $(\mathrm{E})$ catchments for the monitored periods.

\begin{tabular}{|c|c|c|c|c|c|c|c|c|c|c|c|c|c|c|c|c|c|}
\hline \multirow{2}{*}{$\overline{\mathrm{Mes}}$} & & \multicolumn{6}{|c|}{ Año 2008} & \multicolumn{10}{|c|}{ Año 2009} \\
\hline & & 6 & 7 & 8 & 9 & 11 & 12 & 1 & 2 & 3 & 4 & 5 & 6 & 7 & 8 & 9 & \\
\hline Día & & 15 & 10 & 13 & 30 & 8 & 20 & 7 & 13 & 18 & 16 & 20 & 18 & 14 & 15 & 30 & \\
\hline \multicolumn{2}{|c|}{ Período } & & 1 & 2 & 3 & 4 & 5 & 6 & 7 & 8 & 9 & 10 & 11 & 12 & 13 & 14 & $\Sigma$ \\
\hline & $\mathrm{Pp}(\mathrm{mm})$ & & 209 & 231 & 412 & 38 & 32 & 1 & 6 & 4 & 16 & 156 & 237 & 308 & 266 & 234 & 2.149 \\
\hline \multirow{7}{*}{$\mathrm{P} 1$} & Ic (mm) & & 38 & 39 & 43 & 12 & 19 & 3 & 4 & 2 & 7 & 37 & 37 & 43 & 38 & 35 & 357 \\
\hline & $\mathrm{Q}(\mathrm{mm})$ & & 58 & 115 & 174 & 26 & 11 & 2 & 2 & 2 & 2 & 6 & 19 & 92 & 63 & 133 & 705 \\
\hline & Es (mm) & & 17 & 18 & 66 & 1 & 0 & 0 & 0 & 0 & 0 & 2 & 7 & 35 & 20 & 22 & 188 \\
\hline & $\mathrm{Cb}(\mathrm{mm})$ & & 41 & 97 & 109 & 25 & 10 & 2 & 2 & 2 & 2 & 4 & 12 & 56 & 43 & 111 & 517 \\
\hline & $\operatorname{EvTr}(\mathrm{mm})$ & & - & - & - & - & - & - & - & - & - & - & - & - & - & - & 1.405 \\
\hline & $\mathrm{R}(\mathrm{mm})$ & 923 & 941 & 950 & 855 & 770 & 576 & 506 & 483 & 467 & 450 & 555 & 711 & 775 & 959 & 962 & - \\
\hline & St (kg/ha) & & 32 & 41 & 15 & 4 & 3 & 1 & 0 & 0 & 0 & 1 & 41 & 36 & 52 & 11 & 237 \\
\hline \multirow{7}{*}{$\mathrm{P} 2$} & Ic $(\mathrm{mm})$ & & 41 & 33 & 40 & 11 & 16 & 3 & 4 & 3 & 5 & 32 & 39 & 38 & 35 & 37 & 337 \\
\hline & $\mathrm{Q}(\mathrm{mm})$ & & 62 & 113 & 170 & 30 & 15 & 3 & 2 & 2 & 3 & 7 & 11 & 91 & 68 & 129 & 707 \\
\hline & Es (mm) & & 24 & 20 & 59 & 1 & 1 & 0 & 0 & 0 & 0 & 2 & 6 & 33 & 19 & 21 & 185 \\
\hline & $\mathrm{Cb}(\mathrm{mm})$ & & 38 & 93 & 112 & 30 & 14 & 3 & 2 & 2 & 2 & 5 & 5 & 58 & 49 & 108 & 522 \\
\hline & $\operatorname{EvTr}(\mathrm{mm})$ & & - & - & - & - & - & - & - & - & - & - & - & - & - & - & 1.388 \\
\hline & $\mathrm{R}(\mathrm{mm})$ & 912 & 935 & 942 & 838 & 724 & 521 & 458 & 435 & 411 & 406 & 498 & 727 & 782 & 957 & 966 & - \\
\hline & St (kg/ha) & & 96 & 102 & 34 & 6 & 7 & 2 & 1 & 1 & 3 & 8 & 86 & 127 & 127 & 14 & 615 \\
\hline \multirow{7}{*}{ E1 } & Ic $(\mathrm{mm})$ & & 21 & 35 & 32 & 15 & 16 & 2 & 2 & 3 & 1 & 6 & 22 & 12 & 28 & 20 & 215 \\
\hline & $\mathrm{Q}(\mathrm{mm})$ & & 44 & 95 & 127 & 23 & 11 & 3 & 2 & 1 & 2 & 4 & 15 & 45 & 26 & 40 & 439 \\
\hline & Es (mm) & & 17 & 16 & 40 & 1 & 0 & 0 & 0 & 0 & 0 & 2 & 8 & 23 & 10 & 12 & 130 \\
\hline & $\mathrm{Cb}(\mathrm{mm})$ & & 26 & 79 & 87 & 23 & 11 & 3 & 2 & 1 & 1 & 2 & 7 & 22 & 16 & 29 & 309 \\
\hline & $\operatorname{EvTr}(m m)$ & & - & - & - & - & - & - & - & - & - & - & - & - & - & - & 1.630 \\
\hline & $\mathrm{R}(\mathrm{mm})$ & 905 & 928 & 940 & 760 & 712 & 512 & 467 & 426 & 409 & 389 & 525 & 693 & 745 & 980 & 985 & - \\
\hline & St (kg/ha) & & 68 & 22 & 11 & 6 & 2 & 0 & 1 & 2 & 1 & 3 & 17 & 10 & 7 & 2 & 152 \\
\hline \multirow{7}{*}{ E2 } & Ic (mm) & & 31 & 36 & 30 & 18 & 9 & 1 & 2 & 4 & 2 & 13 & 20 & 20 & 31 & 18 & 235 \\
\hline & $\mathrm{Q}(\mathrm{mm})$ & & 56 & 99 & 151 & 28 & 14 & 3 & 3 & 2 & 2 & 6 & 16 & 42 & 28 & 50 & 500 \\
\hline & Es (mm) & & 17 & 12 & 43 & 0 & 1 & 0 & 0 & 0 & 0 & 2 & 8 & 21 & 10 & 11 & 124 \\
\hline & $\mathrm{Cb}(\mathrm{mm})$ & & 39 & 86 & 108 & 28 & 14 & 3 & 3 & 2 & 2 & 4 & 8 & 21 & 18 & 39 & 376 \\
\hline & $\operatorname{EvTr}(\mathrm{mm})$ & & - & - & - & - & - & - & - & - & - & - & - & - & - & - & 1.511 \\
\hline & $\mathrm{R}(\mathrm{mm})$ & 924 & 941 & 951 & 816 & 747 & 542 & 522 & 502 & 494 & 484 & 579 & 778 & 823 & 1.055 & 1.062 & - \\
\hline & St (kg/ha) & & 51 & 15 & 9 & 6 & 2 & 0 & 1 & 1 & 3 & 4 & 14 & 8 & 7 & 2 & 125 \\
\hline
\end{tabular}

Fecha inicio del estudio: 15 junio 2008. -: sin datos. 

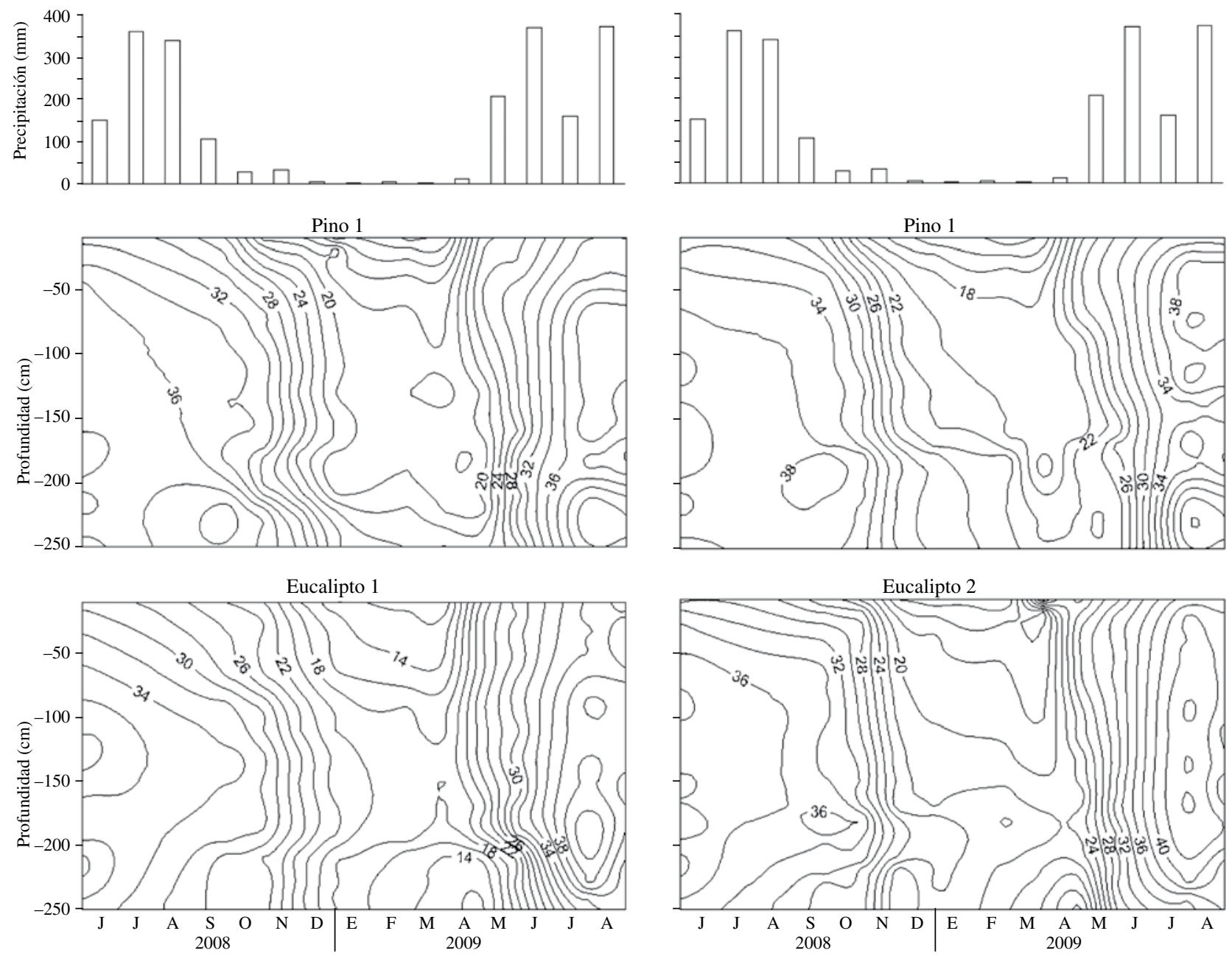

Figura 2. Variación temporal y espacial del contenido de agua edáfica (\% volumétrico) para las cuencas con Pinus radiata (pino) y Eucalyptus globulus (eucalipto).

Spatio-temporal variation of soil water content (\% Vol) of catchments forested with Pinus radiata and Eucalyptus globulus.

tiempo para la zona (INIA 1989). En general, mantienen su distribución temporal, resaltando el déficit pluviométrico durante el verano.

Durante los períodos correspondientes al estío, prácticamente la totalidad de las precipitaciones son interceptadas por la cubierta arbórea. Las diferencias entre las características de las plantaciones de pino y eucalipto son las principales responsables de la desigual pérdida de agua por intercepción. Estas disimilitudes se deben principalmente a que las coníferas tienen una mayor capacidad de retención de agua que las latifoliadas (Crockford y Richardson 2000, Huber y Iroumé 2001, Link et al. 2004, Fleischbein et al. 2005). También influye la menor edad que tienen las plantaciones de eucalipto, que por consiguiente poseen un dosel menos desarrollado. Estas condiciones generan una inferior cobertura del dosel, a pesar de que estas plantaciones tienen una mayor densidad. Por consiguiente, la precipitación neta en las cuencas con eucalipto es significativamente superior a las con pino.
Las pérdidas de agua por intercepción determinadas en el estudio son inferiores a las registradas por Huber y Oyarzún (1984), Huber et al. (1998, 2008), Klaassen et al. (1998), Iroumé y Huber (2000, 2002), Huber y Iroumé (2001), Fleischbein et al. (2005), Deguchi et al. (2006), Cao et al. (2008) en otras localidades de Chile. Ello se debe principalmente a la ubicación altitudinal de las cuencas, que permiten que las plantaciones se encuentren frecuentemente inmersas en neblina o nubes durante los eventos de lluvia. Bajo estas condiciones meteorológicas, los árboles interceptan una parte de la nubosidad para entregarla como un adicional a la precipitación directa. Esta situación subvalora levemente el real valor de las pérdidas de agua por intercepción de estas plantaciones en la zona. Durante algunos eventos de lluvia de poca monta y muy baja intensidad, el aporte adicional de agua por intercepción de la neblina permite que al suelo llegue una cantidad de lluvia que supera la precipitación a campo abierto. De todas maneras, el aporte de agua por estas fuentes en 
comparación a los aportes anuales por precipitación es muy bajo para esta localidad. Estas tendencias coinciden con las obtenidas por otros autores (Klaassen et al. 1998, Holder 2004, Knoblich 2005).

Agua edáfica. La variación espacial y temporal del agua en el suelo es similar en las cuatro cuencas. A partir del período 4, cuando disminuyen las precipitaciones y las condiciones meteorológicas incrementan la intensidad de la evapotranspiración, se registra una disminución en las reservas de agua edáfica. Esta situación se manifiesta en todo el perfil considerado, pero con mayor intensidad en los primeros 1,5 $\mathrm{m}$ de profundidad. En los períodos 8 y 9 , el contenido de agua del suelo en los primeros $20 \mathrm{~cm}$ desciende a valores inferiores a los del punto de marchitez permanente (14\% volumétrico). Este estado se manifiesta especialmente en la componente herbácea de la cubierta vegetal que se extingue por falta de agua.

Al comienzo del estudio, que coincide con la época más lluviosa del año, el contenido de agua del suelo en los primeros 2,5 $\mathrm{m}$ de profundidad es similar para todas las cuencas. Este valor está cercano a la capacidad máxima de retención de agua. Durante el tiempo con menores precipitaciones y mayores consumos de agua por evapotranspiración, las reservas de agua disminuyen para alcanzar un mínimo a principio de otoño. En consecuencia, la cantidad de agua disponible para el desarrollo de los árboles desciende considerablemente. A partir del período 10, que coincide con el inicio de la nueva temporada de invierno y una disminución de la intensidad de la evapotranspiración, comienza una paulatina recarga de agua, que alcanza en todas las cuencas su máximo en septiembre.

La diferencia máxima entre los contenidos extremos de agua del perfil de los suelos, durante todo el período, en las cuencas con pino $(512$ y $560 \mathrm{~mm})$ y eucalipto (596 y $578 \mathrm{~mm}$ ) es reducida. Ello se debe principalmente a que al inicio del estudio el perfil de los suelos de las distintas cuencas está prácticamente saturado y que al final del estío poseen un mínimo que también es similar entre ellos. Esta última situación se logra porque la mayor parte del agua edáfica, especialmente en los primeros $1,5 \mathrm{~m}$, ha sido consumida durante el estío por las plantaciones. En consecuencia, esta condición es una restricción para que las plantaciones puedan alcanzar su mayor potencial de crecimiento durante el verano (Carroll et al. 2000, Zhou et al. 2002, Andreassian et al. 2004). A su vez, es una circunstancia que reduce las diferencias que pueden existir entre los consumos extremos de agua de las dos especies, especialmente durante el período deficitario en precipitaciones (Putuhena y Cordery 2000).

Escorrentía. La variación temporal del caudal de los cuatro efluentes de las cuencas está regulada principalmente por el régimen anual de las precipitaciones, la variación del contenido de agua del suelo y la evapotranspiración (Blume et al. 2007, Mayor et al. 2009).
El agotamiento extremo de la reserva de agua edáfica durante el estío explica por qué el aumento de las precipitaciones de marzo a junio no incrementa en forma similar el caudal de los efluentes. Esta agua se requiere para saturar el perfil del suelo y, con ello, crear las condiciones para generar una escorrentía superficial capaz de incrementar en forma significativa el caudal de los efluentes.

El caudal total de los efluentes de las dos cuencas de pino para todo el período es el mismo, lo que incide en forma idéntica en sus correspondientes coeficientes de escorrentía. Esta situación puede ser consecuencia de la similitud que hay entre ambas cuencas y en las características de las plantaciones forestales.

La cuenca de Eucalipto 1 registra un menor coeficiente de escorrentía en comparación a Eucalipto 2. Esta diferencia podría ser consecuencia de la menor pendiente media de las laderas que tiene la cuenca Eucalipto 1 (Carroll et al. 2000, Blume et al. 2007). Los coeficientes de escorrentía tienen una fuerte variación temporal por estar influidos por las condiciones climáticas, especialmente por el régimen de las precipitaciones, el grado de saturación de los suelos y la evapotranspiración (Blume et al. 2007).

Los efluentes de las cuencas con pino registran para todo el período un mayor caudal que las con eucalipto, a pesar de que al suelo les llega una menor cantidad de agua por tener una superior pérdida por intercepción.

El agua de origen subsuperficial es el principal componente del caudal total en todos los efluentes de las cuencas. Su participación varía según la distribución temporal de las precipitaciones. Durante el invierno, especialmente cuando los suelos están saturados, la escorrentía superficial aumenta su participación relativa en el caudal total en desmedro de la escorrentía subsuperficial. Situación contraria sucede durante el estío, donde la falta de precipitaciones importantes permite que la escorrentía subsuperficial tenga una participación exclusiva (Onda et al. 2001, Blume et al. 2007).

Evapotranspiración. Los montos de evapotranspiración para ambas especies concuerdan con los resultados de otros estudios realizados en Chile que usaron metodologías distintas (Huber y Oyarzún 1985, Oyarzún et al. 1985, Huber et al. 1998, 2008, Huber y Trecaman 2002, 2004).

La evapotranspiración incluidas las pérdidas de agua por intercepción para todo el período son inferiores en las cuencas con pino que en las con eucalipto. En consecuencia, en el presente estudio las plantaciones de eucalipto registran una mayor evapotranspiración. Este resultado se puede deber a las características propias de cada especie y por disponer de una superior cantidad de agua edáfica como consecuencia de las inferiores pérdidas por intercepción.

Durante la época más deficitaria en precipitaciones, la evapotranspiración se sustenta, exclusivamente, de la reserva de agua edáfica. En consecuencia, al final del estío las plantaciones han consumido la mayor parte del 
agua. Esta situación inhibe la evapotranspiración durante el verano, lo que reduce la diferencia potencial de consumo máximo de agua entre las dos especies. En consecuencia, el efecto de la distinta edad, densidad y manejo de las plantaciones no se va a manifestar con todo su rigor en la evapotranspiración. Por consiguiente, la máxima diferencia entre los requerimientos potenciales de agua entre pino y eucalipto se va a presentar recién cuando tengan acceso a toda el agua requerida durante el año y, especialmente, durante el estío.

En consecuencia, la evapotranspiración de las diferentes plantaciones está más bien regulada por la disponibilidad de agua en el suelo durante el período deficitario en precipitaciones que por la capacidad potencial de consumo de cada una de ellas.

Transporte de sedimentos. La cantidad total de sedimentos transportados por los efluentes de las cuencas con pino es mayor que en las con eucalipto. Esta diferencia se puede explicar por el mayor ancho que tienen las zonas de protección de los efluentes de las cuencas con eucalipto. Las características propias de las zonas de protección influyen en la capacidad de retención de sedimentos (Boothroyd et al. 2004, Gomi et al. 2005, Hassan et al. 2005).

Sorprende la mayor cantidad de sedimentos evacuados en la cuenca Pino 2. Este resultado puede ser consecuencia del paso obligado que tiene el efluente por una pequeña zona de inundación que podría aportar un adicional de sedimentos.

La variación temporal de la exportación de sedimentos está influida principalmente por el régimen de las precipitaciones $\mathrm{y}$, por consiguiente, por la intensidad de la escorrentía superficial (Beschta et al. 2000, Gomi et al. 2005, Karwan et al. 2007). El mayor transporte de sedimentos se concentra en los períodos más lluviosos y cuando los suelos están saturados. Esta condición edáfica es importante en la generación del caudal superficial, que incluso puede superar a la de la intensidad de las precipitaciones (Cammeraat 2002, Seeger et al. 2004, Mayor et al. 2009, Turnbull et al. 2010).

Las desiguales características topográficas de las cuencas, las particularidades de cada una de las coberturas del suelo, la distinta intensidad con que están perturbados los suelos debido a intervenciones silviculturales precedentes, las características de las franjas de protección en los cauces y los disímiles montos de la escorrentía superficial de las cuencas, con iguales o distintas cubiertas forestales, son las responsables de la dispar intensidad del transporte de sedimentos (Scott y Lesch 1997, Moore y Wondzell 2005, Gomi et al. 2005, Karwan et al. 2007).

Según la CONAMA (1996), los montos de sedimentos evacuados por los efluentes por unidad de superficie en el presente estudio son considerados como ligeros. Sus totales son superiores a los informados por Oyarzún y Peña (1995), que utilizaron el método de parcelas de erosión para su cuantificación. También superan los valores recopilados por
Gomi et al. (2005) para distintas plantaciones de coníferas ubicadas en el Pacífico noroeste de EE.UU., que oscilan entre uno y $100 \mathrm{~kg} / \mathrm{ha}$. Estas plantaciones están ubicadas en suelos de origen metamórfico que tienen una pluviometría anual que fluctúa entre los 700 y $2.500 \mathrm{~mm}$.

\section{CONCLUSIONES}

Debido a las diferentes características del dosel, las pérdidas de agua por intercepción son mayores en las cuencas cubiertas con pino que en las de eucalipto. La cantidad de agua involucrada en la evapotranspiración en las cuencas de pino es inferior a las de eucalipto. Esto influye en forma inversa en el monto de los caudales de los efluentes de las correspondientes cuencas. La carga de sedimento es superior en las cuencas de pino que en las de eucalipto, situación que podría ser explicada por las distintas características de las zonas de protección de orilla de cauces.

\section{AGRADECIMIENTOS}

El estudio fue financiado con aportes del Fondo Nacional de Investigaciones Científicas y Tecnológicas (FONDECYT) a través del Proyecto $\mathrm{N}^{\circ} 1070218$ y el importante apoyo de la empresa Forestal Mininco S. A. También se agradece a los colaboradores en el proyecto Srs. Rodrigo Bravo y Carlos Torres.

\section{REFERENCIAS}

Andreassian V, C Perrin, C Michel. 2004. Impact of imperfect potential evapotranspiration knowledge on the efficiency and parameters of watershed models. Journal of Hydrology 286:19-35.

Armesto J, D Manuschevich, C Mora, R Smith-Ramírez, A Rozzi, A Abarzúa, P Marquet. 2010. From the Holocene to the Anthropocene: a historical framework for land cover change in southwestern South America in the past 15.000 years. Land Use Policy 27: 148-160.

Beschta R, M Pyles, A Skaugset, C Surfleet. 2000. Peakflow responses to forest practices in the western cascades of Oregon, USA. Journal of Hydrology 233: 102-120.

Blume T, E Zehe, A Bronstert. 2007. Rainfall runoff response, runoff coefficients and baseflow separation. Hydrological Sciences Journal 52: 843-862.

Boothroyd I, J Quinn, E Langer, K Costley y G Steward. 2004. Riparian buffers mitigate effects of pine plantation logging on New Zealand streams. 1. Riparian vegetation structure, stream geomorphology and periphyton. Forest Ecology and Management 194: 199-213.

Brown A, L Zhang, T McMahon, A Western, R Vertessy. 2005. A review of paired catchment studies for determining changes in water yield resulting from alterations in vegetation. Journal of Hidrology 310: 28-61. 
Bruijnzeel LA. 2001. Hydrology of tropical montane cloud forests: a reassessment. Land Use and Water Resources Research 1: 1-18.

Cammeraat L. 2002. A review of two strongly contrasting geomorphological systems within the context of scale. Earth Surface Processes and Landforms 27: 1201-1222.

Carlyle-Moses DE. 2004. Throughfall, stemflow, and canopy interception loss fluxes in a semi-arid Sierra Madre Oriental matorral community. Journal of Arid Environments 58: 181-202.

Cao Y, Z Ouyang, H Zheng, Z Huang, X Wang, H Miao. 2008. Effects of forest plantations on rainfall redistribution and erosion in the red soil region of southern China. Land Degradation and Development 19:321-330.

Carroll C, L Merton, P Burger. 2000. Impact of vegetative cover and slope on runoff, erosion, and water quality for field plots on a range of soil and spoil materials on central Queensland coal mines. Australian Journal of Soil Research 38: 313-327.

CONAMA (Comisión Nacional del Medio Ambiente, CL). 1996. Metodologías para la caracterización de la calidad ambiental. Santiago, Chile. 242 p.

Crockford R, D Richardson. 2000. Partitioning of rainfall into throughfall, stemflow and interception: effect of forest type, ground cover and climate. Hydrological Processes 14: 2903-2920.

Deguchi A, S Hattori, H Park. 2006. The influence of seasonal changes in canopy structure on interception loss: Application of the revised Gash Model. Journal of Hydrology 318: 80102.

Farley K, E Jobbágy, R Jackson. 2005. Effects of afforestation on water yield: a global synthesis with implications for policy. Global Change Biology 11: 1565-1576.

Fleischbein K, W Wilcke, J Boy, C Valarezo, W Zech, K Knoblich. 2005. Rainfall interception in a lower montane forest in Ecuador: effects of canopy properties. Hydrological Processes 19: 1355-1371.

Fuenzalida H. 1971. Climatología de Chile. In Corporación de Fomento de la Producción ed. Geografía Económica de Chile. Santiago, Chile. p. 99-152.

Gomi T, R Moore, M Hassan. 2005. Suspended sediment dynamics in small forest streams of the Pacific Northwest. Journal of the American Water Resources Association (August): 877-898.

Hassan M, M Church, T Lisle, F Brardinoni, L Benda, G Grant. 2005. Sediment transport and channel morphology of small forested streams. Journal of the American Water Resources Association 41: 853-876.

Holder C. 2004. Rainfall interception and fog precipitation in a tropical montane cloud forest in Guatemala. Forest Ecology and Management 190: 373-384.

Hubbart J, T Link, J Gravelle, W Elliot. 2007. Timber harvest impacts on water yield in the Continental/Maritime Hydroclimatic Region of the United States. Forest Science 53(2): 169-180.

Huber A, P Barriga, R Trecaman. 1998. Efecto de la densidad de plantaciones de Eucalyptus nitens sobre el balance hídrico en la zona de Collipulli, IX Región, Chile. Bosque 19(1): 61-69.

Huber A, R Trecaman. 2004. Eficiencia del uso del agua en plantaciones de Pinus radiata en Chile. Bosque 25(3): 33-43.
Huber A, G García. 1999. Importancia de los factores meteorológicos en la transpiración potencial de Pinus radiata. Pyton 65: 143-152.

Huber A, A Iroumé. 2001. Variability of annual rainfall partitioning for different sites and forest covers in Chile. Journal of Hydrology 248: 78-92.

Huber A, C Oyarzún. 1984. Factores reguladores de la intercepción en un bosque adulto de Pinus radiata D. Don. Bosque 6(2): 74-82.

Huber A, R Trecaman. 2002. Efecto de la variabilidad interanual de las precipitaciones sobre el desarrollo de las plantaciones de Pinus radiata (D. Don) en la zona de los arenales, VIII Región, Chile. Bosque 23(2): 43-49.

Huber A, A Iroumé, J Bathurst. 2008. Effect of Pinus radiata plantations on water balance in Chile. Hydrological Processes 22: $142-148$.

Huber A, C Oyarzún, A Ellies. 1985. Balance hídrico en tres plantaciones de Pinus radiata y una pradera. Bosque 6(2): 74-82

INFOR (Instituto Forestal, CL). 2008. Anuario Forestal 2008. Boletín Estadístico 121. Centro de Información Forestal, sede Metropolitana, Santiago, Chile. 169 p.

INIA (Instituto de Investigaciones Agropecuarias, CL). 1989. Mapa agroclimático de Chile. Santiago, Chile. INIA. 221 p.

Iroumé A, O Mayen, A Huber. 2006. Runoff and peak flow responses to timber harvest and forest age in southern Chile. Hydrological Processes 20: 37-50.

Iroumé A, A Huber. 2000. Intercepción de las lluvias por cubiertas de bosques y efecto en los caudales de crecida en una cuenca experimental en Malalcahuello, IX Región Chile. Bosque 21 (1): 45-56.

Iroumé A, A Huber. 2002. Comparison of interception losses in a broadleaved native forest and a Pseudotsuga menziesii (Douglas fir) plantation in the Andes Mountains of southern Chile. Hydrological Processes 16: 2347-2361.

Iroumé A, O Mayen, A Huber. 2006. Runoff and peakflow responses to timber harvest and forest age in southern Chile. Hydrological Processes 20:37-50.

Karwan D, J Gravelle, J Hubbart. 2007. Effects of timber harvest on suspended sediment loads in Mica Creek, Idaho. Forest Science 53(2): 181-188.

Klaassen W, F Boseveld, E De Walter. 1998. Water storage and evaporation as constituents of rainfall interception. Journal of Hydrology (212-213): 36-50.

Knoblich K. 2005. Rainfall interception in a lower montane forest in Ecuador: effects of canopy properties. Hydrological Processes 19: 1355-1371.

Link T, M Unsworth, D Marks. 2004. The dynamics of rainfall interception by a seasonal temperate rainforest. Agricultural and Forest Management 124: 171-191.

May C. 2007. Sediment and wood routing in steep headwater streams: an overview of geomorphic processes and their topographic signatures. Forest Science 53(2): 119-130.

Mayor A, S Bautista, J Bellot. 2009. Factors and interactions controlling infiltration, runoff, and soil loss at the microscale in a patchy Mediterranean semiarid landscape. Earth Surface Processes Landforms 34: 1702-1711.

Megahan W, J King, K Seyesbagheri. 1995. Hydrologic and erosional responses of a granitic watershed to helicopter logging and broadcast burning. Forest Science 41(4): 777-795.

Moore R, S Wondzell. 2005. Physical hydrology and the effects of forest harvesting in the Pacific northwest: a review. Journal of the American Water Resources Association 41:763-784. 
Onda Y, Y Komatsu, M Tsujimura, J Fujihara. 2001. The role of subsurface runoff through bedrock on storm flow generation. Hydrological Processes 15: 1693-1706.

Oyarzún C. 1993. Evaluación del modelo U.S.L.E. para predecir pérdidas de suelo en áreas forestadas de la cuenca del río Biobío. Bosque 14(1): 45-54.

Oyarzún C, L Peña. 1995. Soil erosion and overland flow in forested areas with pine plantations at coastal mountain range, central Chile. Hydrological Processes 9: 111-118.

Oyarzún C, A Huber, S Vásquez. 1985. Balance hídrico en tres plantaciones de Pinus radiata. I: Redistribución de las precipitaciones. Bosque 6 (1): 3-13.

Pizarro T, H Cuitiño. 1999. Evaluación cuantitativa de la erosión hídrica superficial en suelos desnudos de la Pre-Cordillera Andina y Valle Central de la VII Región. In VI Jornadas del CONAPHI. Chile.

Putuhena W, I Cordery. 2000. Some hydrological effects of changing forest cover from eucalypts to Pinus radiata. Agricultural and Forest Meteorology 100:59-72.

Rehg K, A Packman, J Ren. 2005. Effects of suspended sediment characteristics and bed sediment transport on streambed clogging. Hydrological Processes 19:413-427.

Robinson M, A Cognard-Plancqb, C Cosandey, J David, P Durand, H Führer, R Hall, M Hendriques, V Marc, R McCarthy, M McDonnell, C Martin, T Nisbet, P O'Dea, M Rodgers, A Zollner. 2003. Studies of the impact of forests on peakflows and baseflows: a European perspective. Forest Ecology and Management 186: 85-97.

Schlatter J, R Grez, V Gerding. 2003. Manual para el reconocimiento de suelos. $3^{\text {a }}$ Edición. Valdivia, Chile, Universidad Austral de Chile. 114 p.
Scott D, F Prinsloo. 2008. Longer-term effects of pine and eucalypt plantations on streamflow. Water Resource Research 44: 1-8.

Scott D, W Lesch. 1997. Streamflow responses to afforestation with Eucalyptus grandis and Pinus patula and to felling in the Mokobulaan experimental catchments, South Africa. Journal of Hydrology 199: 360-377.

Seeger M, M Errea, S Beguería, J Arnáez, C Martí, J García-Ruiz. 2004. Catchment soil moisture and rainfall characteristics as determinant factors for discharge/suspended sediment hysteretic loops in small headwater catchments in the Spanish Pyrenees. Journal of Hydrology 288: 299-311.

Steubing L, A Fangmeier. 2001. Pflanzan-ökologisches Praktikum. Stuttgart, Alemania. Eugen Ulmer. 205 p.

Turnbull L, J Wainwright, R Brazier. 2010. Changes in hydrology and erosion over a transition from grassland to shrubland. Hydrological Processes 24: 393-414.

Van Dijk A, L Bruijnzeel. 2001. Modelling rainfall interception by vegetation of variable density using an adapted analytical model. Part 1. Model description. Journal of Hydrology 247:230-238.

Vega JA, C Fernández, T Fonturbel. 2005. Throughfall, runoff and soil erosion after prescribed burning in gorse shrubland in Galicia (NW Spain). Land Degradation \& Development 16: 37-51.

Zhou G, J Morris, J Yan, Y Zy, S Peng. 2002. Hydrological impacts of reforestation with eucalypts and indigenous species: a case study in southern China. Forest Ecology and Management 167:209-222. 